\title{
Lidil
}

Revue de linguistique et de didactique des langues

$37 \mid 2008$

Syntaxe et sémantique des prédicats

\section{Opérations de prédication et de détermination}

\author{
Jean-Pierre Desclés
}

\section{OpenEdition}

Journals

Édition électronique

URL : http://journals.openedition.org/lidil/2689

DOI : 10.4000/lidil.2689

ISSN : 1960-6052

\section{Éditeur}

UGA Éditions/Université Grenoble Alpes

\section{Édition imprimée}

Date de publication : 1 juin 2008

Pagination : 61-98

ISBN : 978-2-84310-124-3

ISSN : $1146-6480$

Référence électronique

Jean-Pierre Desclés, «Opérations de prédication et de détermination », Lidil [En ligne], 37 | 2008, mis en ligne le 01 septembre 2009, consulté le 01 mai 2019. URL : http://journals.openedition.org/ lidil/2689 ; DOI : 10.4000/lidil.2689

(c) Lidil 


\title{
OPÉRATIONS DE PRÉDICATION ET DE DÉTERMINATION
}

\author{
JEAN-PIERRE DESCLÉS * \\ LaLIC, Paris-Sorbonne
}

\begin{abstract}
En partant d'un rappel des Grammaires Catégorielles, l'auteur présente rapidement le formalisme applicatif où l'opération de base est une application d'un opérateur à un opérande. Il introduit les types fonctionnels de Church pour fournir une explicitation des différentes opérations mises en œuvre par les langues naturelles : prédication, détermination, transposition. La notion générale d'opérateur doit être distinguée de celles d'opérateurs plus spécifiques comme les prédicats, les opérateurs de détermination, de transposition... Un prédicat est un opérateur dont le but est la construction d'une proposition à partir de ses différents arguments. Un opérateur de détermination a pour but d'apporter une information plus spécifique à un terme nominal, à un prédicat, à une phrase entière... Les types fonctionnels permettent de préciser la description de ces opérateurs. La décomposition d'une phrase entre un Prédicat (logique) et un Sujet (logique) est introduite en considérant que la copule «être» doit être analysée comme un opérateur constructeur d'un Prédicat à partir de termes, plus ou moins déterminés, de déterminants (des adjectifs par exemple)... Plusieurs linguistes (Harris, Shaumyan, Culioli, Berrendonner, Le Guern, Pottie...) font appel explicitement, dans leurs analyses, à des opérateurs linguistiques. Cet article a pour objet d'apporter une certaine clarification aux usages des opérateurs en logique et en linguistique, afin de mieux étudier le statut de la prédication. Selon l'auteur, il serait nécessaire de mieux articuler la linguistique avec la logique et la cognition afin d'étudier plus profondément la nature même du langage qui expriment des représentations cognitives et mettent en cuvre des raisonnements logiques, par le biais de constructions sémiotiques spécifiques aux langues naturelles.
\end{abstract}

\section{ABSTRACT}

Starting with Categorial Grammars, the author presents applicative formalism, where the basic operation is the application of an

\footnotetext{
* Université Paris-Sorbonne, LaLIC.
} 


\begin{abstract}
operator to an operand, using Church's functional types to explain the different operations in natural languages: predication, determination, transposition operations (type raising). The general notion of operator is to be distinguished from more particular operators characterized by specific functional types : predicates, determination and transposition operators... A predicate is used to construct a proposition (or a predicative relation) from arguments. A determination operator is used to bring more specific information to a term or a predicate or a sentence. The functional types are tools to name the different operators. When a sentence is broken down into a (logical) Predicate and a (logical) Subject, the copule "be" is analyzed as a binary operator that builds a Predicate from determinants, (adjectives, for example)... Some linguists (Harris, Shaumyan, Le Guern, Culioli, Pottier...) have worked explicitly with operators. This article is a conceptual analysis of some operators used in linguistics and logics to highlight the notion of predication. According to the author, it is necessary to articulate linguistics, logics and cognition to study in depth the nature of Language which expresses cognitive representations and reasoning schemata by means of semiotic configurations.
\end{abstract}

Le formalisme des types des Grammaires catégorielles $(G C)$, simples et étendues (Moortgat, 1988; Biskri et Desclés, 1997, 2006; Steedman 2000), et sa généralisation polystratale avec d'un côté, la Grammaire applicative universelle (GAU) (Shaumyan, 1965, 1987; Desclés et al., 1985) et d'un autre côté, la Grammaire applicative et cognitive $(G A \& C)$ (Desclés, 1990,2004) constituent des modèles linguistiques pour étudier la diversité des opérations constitutives des phrases et, de façon plus complexe, des énoncés lorsqu'on introduit les conditions d'énonciation dans les représentations métalinguistiques (Desclés, 1976, 2006). En effet, ce formalisme assigne des types aux unités linguistiques, les types étant engendrés à partir de types de base; par un véritable calcul sur les types, au moyen de quelques règles, se met en place l'analyse des principales catégories (morphologiques, syntaxiques et, en partie, sémantiques) des langues. Remarquons que le formalisme des types fonctionnels sous-jacent aux Grammaires catégorielles et aux Grammaires applicatives est étroitement lié au formalisme applicatif ${ }^{1}$, ou fonctionnel, qui

1. Les types dont nous parlons ici sont tous des types fonctionnels 
prend pour opération primitive la construction d'un résultat par application d'un opérateur à un opérande. Lorsque sont pris en compte différents types d'opérateurs, l'application n'est pas libre, ce qui conduit à introduire des conditions restrictives sur les possibilités de l'application d'un opérateur ${ }^{2}$.

Un type syntaxique est la spécification fonctionnelle d'une catégorie dont les instances sont une classe paradigmatique d'unités - donc substituables les unes aux autres - ayant un même comportement syntagmatique, ce qui revient à dire que d'une part, toutes les instances de la classe caractérisée par un type syntaxique ont exactement les mêmes capacités combinatoires que les autres unités d'un autre type syntaxique et d'autre part, que l'examen de la forme du type permet d'en analyser sa complexité, c'est-à-dire sa genèse à partir des types de base et son insertion dans le réseau structuré des catégories syntaxiques.

Dans le cadre plus global de la Grammaire applicative et cognitive ( $G A \& C)$, les types syntaxiques, étudiés spécifiquement par les Grammaires catégorielles $(G C)$, entretiennent des relations avec les types morphologiques (responsables de la décomposition analytique des mots), avec les types grammaticaux (responsables des principales fonctions grammaticales, comme «agent», «patient», ou encore les «types d'aspectualité ») et les types logiques et enfin avec les types sémantiques et cognitifs; ces relations imposent différents niveaux d'analyse du langage. Il nous est apparu qu'il fallait concevoir au moins trois niveaux d'analyse: (i) un niveau des configurations morphosyntaxiques spécifiques à chacune des langues; (ii) un niveau plus général des agencements logicogrammaticaux; (iii) un niveau des représentations sémantico-

d'opérateurs. Les types des opérateurs spécifient à la fois le type (ou la catégorie) des opérandes sur lesquels les opérateurs peuvent agir et le type des résultats construits. II ne faut donc pas confondre ces types fonctionnels (Church, 1940) avec les types hiérarchisés des classes de Russell.

2. Un système applicatif de représentations typées considère un langage de types (fonctionnels) comme étant un système de spécifications formelles des instances des catégories associées, ce qui conduit à l'isomorphisme de Curry-Howard (Hindley \& Seldin, 1986). 
cognitives ${ }^{3}$. Pour articuler ces niveaux entre eux, il est nécessaire de concevoir en même temps une architecture cognitive et computationnelle qui établit des relations entre ces niveaux, sous la forme de processus de décomposition, ou de synthèse, d'une unité d'un certain niveau en fonction d'unités d'autres niveaux (Desclés, 1990, 2003, 2004).

Nous allons rappeler le cadre général des Grammaires catégorielles simples du genre A-B (pour Adjukiewicz et BarHillel) puis nous développerons quelques remarques en nous situant plus particulièrement au niveau des analyses logicogrammaticales, de façon à mieux préciser les opérations de prédication parmi d'autres types d'opérations nécessaires aux constructions des agencements sémiotiques observés dans la diversité des langues, comme par exemple, les opérations de détermination. Cela nous conduira à mieux préciser et à étudier les rapports, souvent conflictuels, entre les analyses logiques et les analyses linguistiques des langues naturelles, puisque les réflexions logico-grammaticales sur le langage (le «logos») prennent naissance, au moins en Occident, dès l'aube de la pensée réflexive, avec le «Poème» de Parménide, puis avec Aristote et les Stoïciens, pour se poursuivre, jusqu'à notre époque, avec la philosophie du langage de W.V.O. Quine (1970) et les travaux formels de R. Montague (1974), en passant par les recherches médiévales, et, plus tard, la Logique et la Grammaire de Port Royal...

\section{Grammaires catégorielles}

Dans une Grammaire catégorielle $(G C)$ du genre A-B (Oehrle et al., 1988), il y a deux sortes d'unités de base, d'où les types

3. Comme de nombreux modèles - par exemple le modèle «senstextes» de I. Mel'čuk (1987) - la Grammaire applicative et cognitive $(G A \& C)$ comprend plusieurs niveaux d'analyse; chaque niveau a une certaine autonomie, il est organisé avec des types spécifiques; ces niveaux sont articulés entre eux par des processus de décomposition analytique (dans une démarche ascendante ou bottom $u p$ ), ou d'intégration synthétique (dans une démarche descendante ou top down) (Desclés, 1990, 2003). 
de base: le type des noms et le type des phrases, que 1'on désigne par les symboles respectifs ' $N$ ' et ' $S$ '. Personnellement, nous y adjoignons, sans en argumenter ici les raisons, le type ' $N$ '', qui correspond aux syntagmes nominaux complets. Ces trois types de base ' $N$ ', ' $N$ *' et ' $\mathrm{S}$ ' se voient associer des classes d'instances (respectivement des noms, des syntagmes nominaux complets, des phrases) construites dynamiquement. Des opérations sur les types, désignées par respectivement ' $\%$ ' et ' ' engendrent tous les types dérivés. Nous avons les deux règles suivantes qui caractérisent l'ensemble structuré des types syntaxiques:

(i) Les types syntaxiques de base sont des types

syntaxiques;

(ii) SI ' $\mathrm{X}$ 'et ' $\mathrm{Y}$ 'sont des types syntaxiques Alors ' $\mathrm{X} / \mathrm{Y}$ 'et ' $\mathrm{X} \backslash \mathrm{Y}$ 'sont des types syntaxiques.

Les types ' $\mathrm{X} / \mathrm{Y}$ ' et ' $\mathrm{X} \backslash \mathrm{Y}$ ' se voient associer des classes d'unités linguistiques (des instances) qui fonctionnent comme des opérateurs applicables, avec des restrictions, à des opérandes : une unité syntaxique ' $\mathrm{u}$ ' de type ' $\mathrm{X} / \mathrm{Y}$ ' (lire: « $\mathrm{X}$ sur $Y »)$ est un opérateur qui s'applique seulement à un opérande ' $v$ ' de type ' $Y$ ', positionné sur la chaine syntagmatique immédiatement à la droite de l'opérateur ' $u$ ', pour construire une nouvelle unité ' $u v$ ' de type ' $X$ '; une unité syntaxique ' $u$ ' de type ' $X \backslash Y$ ' (lire: "X sous $Y$ ») est un opérateur qui s'applique seulement à un opérande ' $\mathrm{V}$ ' de type ' $\mathrm{Y}$ ', positionné sur la chaine syntagmatique immédiatement à la gauche de l'opérateur ' $\mathrm{u}$ ', pour construire une nouvelle unité 'vu' de type ' $\mathrm{X}$ ' 4 . Les différentes catégories syntaxiques sont donc clairement caractérisées par un type syntaxique spécifique. Donnons les types syntaxiques de quelques catégories syntaxiques usuelles :

' $S N^{*}$ ' identifie la catégorie des verbes intransitifs (mono-actanciels);

' $\left(\mathrm{S} \backslash \mathrm{N}^{*}\right) / \mathrm{N}^{*}$ ' identifie la catégorie des verbes transitifs (bi-actanciels);

' $N^{*} / N^{\prime} \quad$ identifie la catégorie des articles;

4. Nous adoptons les notations de M. Steedman (2000). Pour une introduction aux grammaires catégorielles, voir, par exemple, l'excellent manuel de J. Lyons (1970:175-178); voir également Lambek (1961); Oehrle et al. (1988), Desclés (2000). 


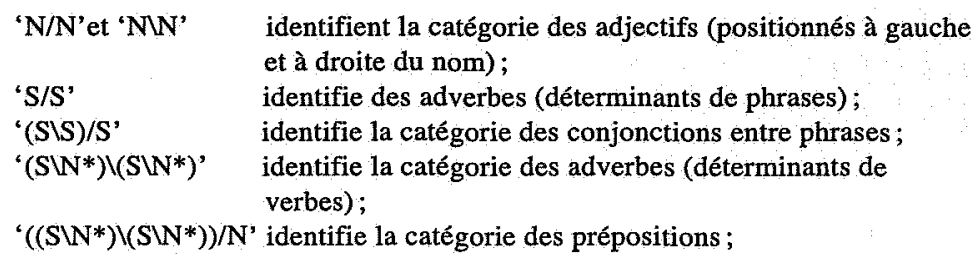

L'assignation d'un type ' $X$ ' à une unité linguistique ' $u$ ' est notée $[\mathrm{u}: \mathrm{X}]$. L'analyse syntaxique d'une phrase revient alors à effectuer un calcul sur les types: chaque unité linguistique (par exemple un mot) ayant un type assigné dans une séquence concaténée soumise à l'analyse syntaxique, le calcul syntaxique, selon l'expression de Lambek (1961), revient à vérifier que le type assigné à la séquence totale est bien de type ' $S$,' en appliquant uniquement les deux règles déductives (à droite et à gauche) suivantes ${ }^{5}$ :
$[\mathrm{u}: \mathrm{X} / \mathrm{Y}]+[\mathrm{v}: \mathrm{Y}]$
$[\mathrm{v}: \mathrm{Y}]+[\mathrm{u}: \mathrm{X} \backslash \mathrm{Y}]$
[uv: $\mathrm{X}]$
[vu: $\mathrm{X}]$

À titre d'exemple illustratif, donnons l'arbre syntaxique construit par une Grammaire $\mathrm{AB}$, de la phrase Le jeune poulain court vite vers sa mère:

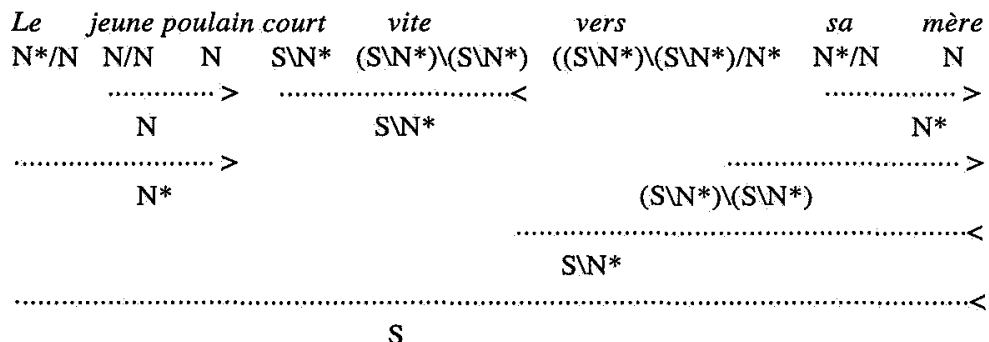

La table des types syntaxiques (et les catégories associées) montre vite que les catégories syntaxiques traditionnelles ne sont pas en bijection avec les types syntaxiques. En effet, pour ne prendre qu'un exemple, la catégorie des adverbes n'est pas homogène puisque plusieurs types d'adverbes peuvent être définis: les modificateurs de verbes (intransitifs,

5. Le symbole '+' désigne, ici, l'opérateur de concaténation. 
transitifs...), ceux qui modifient récursivement d'autres adverbes (comme très dans il court très, très, très... vite), les modificateurs d'adjectifs (très dans très lent), les modificateurs de phrases entières (malheureusement...). On peut montrer également que la catégorie des prépositions n'est pas non plus homogène: certaines prépositions, comme de dans le livre de Pierre, établissent une relation entre deux noms, d'autres prépositions, comme à (aller à Paris), sur (monter sur le toit), de (sortir de la ville)... construisent une relation entre un nom et un verbe. Les types syntaxiques et le calcul qu'elles engendrent donnent donc une analyse beaucoup plus fine que les catégories traditionnelles. Inversement, comme nous allons le montrer par la suite, le regroupement de catégories traditionnelles à l'aide de schémas communs de types, en introduisant des variables de types, permet de dégager des fonctions syntaxiques générales que les noms attribués aux catégories traditionnelles ont complètement obscurcies. Ainsi, les fonctions importantes de prédication et de détermination sont réparties entre des catégories syntaxiques sans liens transparents alors que l'analyse formelle des types syntaxiques nous amène à certains regroupements, par exemple entre verbes intransitifs, transitifs, syntagmes verbaux qui réalisent la fonction grammaticale de prédication, ou entre adjectifs et adverbes qui réalisent une fonction grammaticale de détermination.

Par ailleurs, les rôles d'opérateurs et d'opérandes, qui sont explicitement représentés et notés par les types syntaxiques, peuvent parfois être arbitraires puisque, formellement du moins, dans certains cas, les rôles peuvent s'inverser. Ainsi, dans l'analyse de Luc dort, le verbe dort est analysé comme opérateur qui s'applique à son opérande $L u c$ mais il est également possible de considérer $L u c$ comme un opérateur (ayant alors pour type assigné : ' $\mathrm{S} /\left(\mathrm{S} \mathrm{N}^{*}\right)^{\prime}$ ') qui s'applique à son opérande verbal (toujours de type ' $S \mathrm{NW}^{*}$ ') pour former une unité linguistique de type ' $S$ ' ${ }^{6}$. Remarquons bien que les règles qui

6. Ce changement de type a été introduit à propos des pronoms par J. Lambek (Oehrle et al., 1988). Il a été systématiquement utilisé par R. Montague (1974) dans son analyse des syntagmes nominaux 
opèrent sur les types dans les Grammaires Catégorielles expriment deux notions: d'une part, l'application d'une unité opérateur d'un certain type à une unité opérande d'un certain type, l'opérande étant appelé par le type de l'opérateur, et d'autre part, un ordre syntagmatique (de juxtaposition) entre l'opérateur et l'opérande attendu sur la chaine syntagmatique à la droite, ou à la gauche, de l'opérateur.

Nous allons désormais faire abstraction de cet ordre syntagmatique pour mieux dégager les rôles syntaxiques et grammaticaux des unités linguistiques dans les agencements syntaxiques. Pour cela, nous allons changer de niveau ${ }^{7}$ et considérer les types fonctionnels engendrés à partir des deux types de base ' $p$ ' (pour proposition, ou construction prédicative) et ' $t$ ' (pour terme nominal). Les types fonctionnels sont alors engendrés à partir de ces deux types de base au moyen d'un opérateur préfixé ' $O$ ', constructeur des types dérivés, et des deux règles suivantes:

(i) Les types de base ' $p$ ' et 't' sont des types fonctionnels ;

(ii) SI ' $x$ ' et ' $y$ ' sont des types fonctionnels, ALORS 'Oyx'

est un type fonctionnel.

Puisque les règles introduisent un mécanisme récursif, nous pouvons abréger la notation:

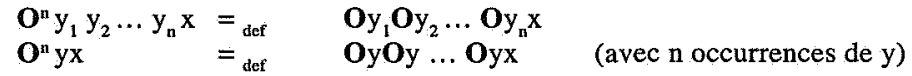

L'interprétation d'une instance ' $U$ 'qui se voit assigner le type assigné 'Oyx'(ce que l'on note: [U: Oyx]) est, comme précédemment, la suivante: ' $U$ ' est un opérateur qui, lorsqu'il s'applique à un opérande ' $V$ ' de type ' $y$ ', construit dynamiquement un résultat de type ' $x$ '. Nous en déduisons la règle applicative et sa généralisation ${ }^{8}$ :

quantifiés. Une règle générale de changement de types et une règle de composition fonctionnelle des types sont à la source des grammaires catégorielles étendues (Moorgat, 1988 ; Steedman, 2000 ; Biskri et Desclés, 1997, 2006 b).

7. Cela revient à passer du niveau des configurations morpho-syntaxiques aux représentations applicatives logico-grammaticales où sont clairement exprimés les opérateurs et les opérandes.

8. Dans ce cas, nous avons n opérations successives d'application qui 


\begin{tabular}{|c|c|}
\hline$[\mathrm{U}: \mathbf{O y x}],[\mathrm{V}: \mathrm{y}]$ & {$\left[U: O^{n} y_{1} y_{2} \ldots y_{n} x\right],\left[V_{1}: y_{1}\right],\left[V_{2}: y_{2}\right], \ldots,\left[V_{n}: y_{n}\right]$} \\
\hline [UV: $\mathrm{x}]$ & {$\left[\mathrm{UV}_{1} \mathrm{~V}_{2} \ldots \mathrm{V}_{\mathrm{n}}: \mathrm{x}\right]$} \\
\hline
\end{tabular}

\section{Principales opérations grammaticales}

Nous pouvons maintenant dégager les principaux rôles grammaticaux et les caractériser par des types fonctionnels et des schémas de types. Le rôle grammatical des prédicats $\mathbf{n}$-aires est toujours exprimé par les schémas de type:

$O^{n} t p$ et $O^{n} y_{1} y_{2} \ldots y_{n} p$ (où chaque ' $y_{1}$ 'est soit ' $t$ ', soit ' $p$ ') ${ }^{9}$ Le premier schéma correspond aux prédicats n-aires (prédicat unaire : ... dort, prédicat binaire: ... regarde ..., prédicat ternaire: ... pose... sur...) qui, en tant qu'opérateurs, s'appliquent uniquement à des termes; le second correspond à des prédicats (comme pense..., voit...) qui acceptent une proposition de type ' $p$ ', comme étant l'un des arguments nécessaires pour la construction d'une proposition construite avec ces prédicats. La fonction (au sens, ici, de but à accomplir) d'un prédicat est donc celle d'un opérateur qui doit construire une proposition $^{10}$ à partir d'un certain nombre d'arguments (ses

construisent progressivement le résultat final, c'est-à-dire la relation prédicative. L'expression du résultat est préfixée au sens suivant: l'opérateur est toujours positionné devant son opérande (ou la succession de ses arguments). Avec cette convention sémiotique, l'expression ' $U V_{1} V_{2} \ldots V_{n}$ 'désigne l'expression applicative suivante: ' $\left(\ldots\left(\left(U_{1}\right)\right.\right.$ $\left.\left.\mathrm{V}_{2}\right) \ldots\right) \mathrm{V}_{\mathrm{n}}$ '.

9. Cette caractérisation des types des prédicats est une condition nécessaire mais elle pas suffisante. En effet, les opérateurs de type OpOpp ne sont pas considérés traditionnellement comme des prédicats (aussi bien en logique qu'en linguistique) mais plutôt comme des connecteurs (comme $e t$, ou implique) reliant des unités du même type (ici, des propositions) pour construire une unité encore du même type. Un opérateur de type Opp (par exemple le type des modaux, dont l'opérateur de négation, comme il est possible que ou peut-être) ne sont pas considérés comme des prédicats mais simplement comme des opérateurs opérant sur des constructions prédicatives déjà constituées. Sur les notions d'opérateur et de prédicat, voir Harris, 1968, 1976, 1982; Shaumyan, 1987; Martin, 1992; Le Guern, 2003.

10. Dans une théorie de l'énonciation, nous distinguons 
opérandes) qui sont soit uniquement des termes et, dans ce cas, le prédicat est dit prédicat du premier ordre, soit des termes et des propositions emboitées considérées également comme des arguments du prédicat. L'opération de prédication consiste alors à appliquer un prédicat à ses arguments (des termes ou des propositions) dans le but de construire une proposition, c'est-à-dire une construction prédicative susceptible d'avoir une valeur de vérité et de dénoter des situations (réalisées, non réalisées ou dont la réalisation est simplement envisagée selon les conditions d'énonciation) ${ }^{11}$. Pour la clarté de la discussion ${ }^{12}$, nous proposons de distinguer d'un côté, les prédicats lexicaux $\mathrm{n}$-aire $\mathrm{P}_{\mathrm{n}}-$ (quelque chose) tombe; (quelqu'un) tue (quelqu'un); (quelqu'un) donne (quelque chose) à (quelqu'un) - et d'un autre côté, un Prédicat (logique) qui s'oppose, dans la décomposition de la construction prédicative, au Sujet (logique) ${ }^{13}$.

explicitement la proposition de l'énoncé qui l'énonce au moyen d'opérateurs d'énonciation de "prise en charge»(Culioli, 1990, 1992, 2002; Desclés, 1976).

11. Pour Frege, et à partir de lui en logique classique, la référence d'une proposition est une valeur de vérité. Pour l'analyse des langues, il parait plus adéquat de considérer qu'une proposition a pour valeur référentielle une situation (du monde perçu ou d'un monde représenté, réel ou imaginaire). Dans le cas d'une phrase simple, la proposition coïncide souvent avec elle mais certaines phrases complexes contiennent plusieurs propositions qualifiées parfois de «clauses». En se plaçant dans le cadre d'une théorie énonciative, une construction prédicative, exprimée par une proposition, est «prise en charge» par un énonciateur; dans ce cas, la proposition emboîtée devient opérande de l'opération de «prise en charge», le résultat construisant l'énoncé. Nous devons donc distinguer, explicitement, les concepts de «phrase »/《énoncé »/《proposition » (ou relation prédicative).

12. Pour comparer différentes approches théoriques et, en particulier différentes utilisations de mêmes concepts par des disciplines différentes, nous devons introduire certaines distinctions nécessaires qui permettent ainsi de transcender les différents usages d'un même concept, en exhibant toutefois les propriétés communes. L'analyse épistémologique de l'interdisciplinarité impose cette hygiène conceptuelle.

13. La notion de «Prédicat logique » (en intégrant toutefois la copule comme un élément constitutif) retrouve la distinction de la logique ancienne qui remonte sans doute à Aristote et décompose la proposition en deux grandes composantes. Certains auteurs intègrent la copule est 
Ainsi, nous avons la construction de la proposition, exprimée avec une notation préfixée donne à Paul un-livre Luc (en français: Luc donne un livre à Paul), effectuée en trois temps, avec le prédicat lexical donne... à:

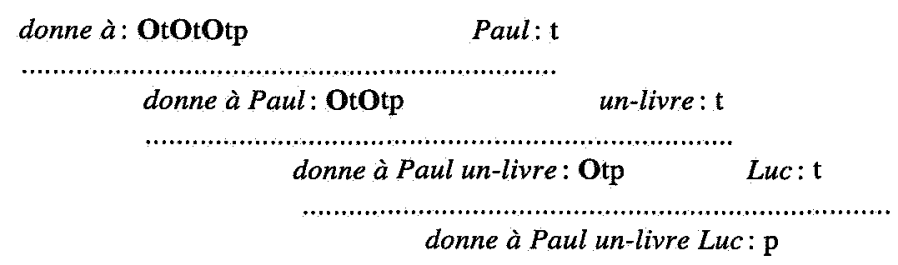

Dans cette construction propositionnelle, le Prédicat (logique) 'donne à Paul un-livre' s'oppose au Sujet (logique) Luc. Avec cette distinction prédicat lexical/Prédicat logique, on exprime la vieille opposition (logique) dans l'analyse propositionnelle des phrases Socrate est un homme; Les hommes sont mortels où 'est un homme' et 'sont mortels' sont des Prédicats logiques.

Le statut de «copule» est souvent attribué à est; son rôle revient alors à mettre en relation d'une part, un «élément prédicatif », c'est-à-dire une unité qui est, par nature, incomplète et attend des arguments pour construire une proposition, et d'autre part, un Sujet - souvent un sujet syntaxique mais pas toujours. Pour remplir cette fonction de mise en relation, la copule est devient un opérateur de mise en relation (appelé alors relateur) qui construit un Prédicat (logique) appliqué ensuite au Sujet.

Souvent, dans les textes linguistiques, ce qui est appelé «prédicat» est une unité linguistique, désignée ici par «élément prédicatif», qui intègre parfois la «copule». On

\footnotetext{
dans la composante du Prédicat logique, d'autres analysent est comme un simple relateur de liaison qui n'aurait aucune signification. Il est certain que certains prédicats lexicaux sont également des Prédicats logiques, mais, évidemment, certains Prédicats logiques ne sont pas des prédicats lexicaux. Si les prédicats lexicaux ayant pour arguments uniquement des termes ont des types de la forme $\mathbf{O}^{\mathrm{n}} \mathrm{tp}$, un Prédicat logique est unaire et a pour type Otp. La non distinction entre prédicat et Prédicat est souvent source de confusions oiseuses et mal cadrées. La convention typographique qui distingue «prédicat» (lexical) et «Prédicat » (logique) est commode pour ce genre de discussions.
} 
confond souvent dans ces textes des unités linguistiques comme homme, est homme, est un homme ou, encore, libre et est libre... Du point de vue linguistique et logique, il devient alors difficile d'étudier les différentes valeurs de la copule est (identification, appartenance à et inclusion dans une classe, ingrédience méréologique - partie d'un tout -, repérage de localisation...) et de distinguer les rôles constructifs des noms communs, des adjectifs et des verbes intransitifs qui ont, bien entendu, des propriétés différentielles, à la fois logiques, sémantiques, morphologiques et syntaxiques. Dans la conception que nous défendons, le type de l'opérateur 'est' devient donc: 'OtOtp' dans la phrase Napoléon est le vainqueur d'Austerlitz, avec la valeur d'identification de l'occurrence de est; OtOtp dans la phrase Napoléon est un homme et 'OOttOtp' dans la phrase Alfred est intelligent, où, dans ces deux phrases, l'appartenance à une classe est la valeur de l'occurrence de est ;'OOttOtp' dans la phrase Les hommes sont mortels, où l'occurrence de la est a la valeur d'inclusion entre classes ; 'OOOtpOtpOtp' dans Alfred est devant.

Devant la diversité des types et des significations assignables selon les occurrences de est, on pourrait être amené à affirmer que cette unité linguistique n'aurait par elle-même aucune signification intrinsèque ${ }^{14}$ ayant seulement un rôle incolore de simple mise en relation. Or, pour nous, est possède une signification complexe, avec un invariant sémantique, que nous ne présentons pas et ne discuterons pas $\mathbf{i c i}^{15}$, et avec un rôle constructif clair: construire un Prédicat à partir d'éléments prédicatifs, c'est-à-dire d'opérateurs précis ${ }^{16}$, qui ne sont pas pour autant des prédicats. En introduisant une

14. Certains auteurs le font explicitement. Pour des éléments de discussions, voir Le Guern (2003 : 116-123).

15. Cet invariant est un schème binaire de repérage abstrait, susceptible de prendre plusieurs valeurs selon la nature des éléments mis en relation. Ce schème est lié à l'opérateur «epsilon » - et son opérateur dual - de Culioli et al. (1970) et Culioli (1990) et à l'analyse, proposée, entre autres, par E. Benveniste, de «être» et «avoir» dans les langues.

16. Ces unités prédicatives sont en soi incomplètes. Pour reprendre la terminologie de Frege, elles sont «non saturées». Ce sont nécessairement des syncatégorèmes. 
variable ' $\mathrm{x}$ ' de type, le schéma de type assigné à l'opérateur

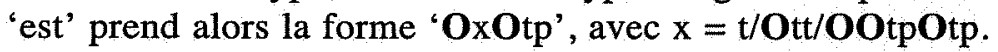
Un tel schéma de type indique clairement que 'est' doit être analysé comme un constructeur de Prédicat et également - le type fonctionnel le signifie explicitement - comme un opérateur de mise en relation d'un élément prédicatif (de type ' $x$ ') avec un terme pour construire une proposition. La formalisation à l'aide des types fonctionnels, des opérateurs et avec une distinction établie entre prédicats (lexicaux), unités prédicatives et Prédicats permet de débrouiller une situation qui est souvent fort confuse dans la littérature consacrée à l'analyse de la prédication.

La fonction grammaticale de détermination est caractérisée par le schéma de type: $O x x$, où $x$ est une variable de type. Un déterminant est une instance de ce type; c'est donc un opérateur qui s'applique à un opérande de type ' $x$ ' pour construire une nouvelle unité (plus complexe) de même type ' $\mathrm{X}$ '. Ainsi, un adjectif, un complément de nom, une relative, ont le type Ott, leurs fonctions sont déterminatives puisqu'elles apportent une nouvelle information à un terme (un beau livre, le livre de Pierre, le livre qui est dans la bibliothèque...). La fonction de connexion est caractérisée par le schéma de type: $\mathrm{O}^{2} \mathrm{xx}={ }_{\text {def }} \mathrm{OxO} O \mathrm{xx}$.

Nous donnons dans le tableau suivant quelques schémas de type qui correspondent aux fonctions et opérations grammaticales que l'on retrouve dans la plupart des langues naturelles.

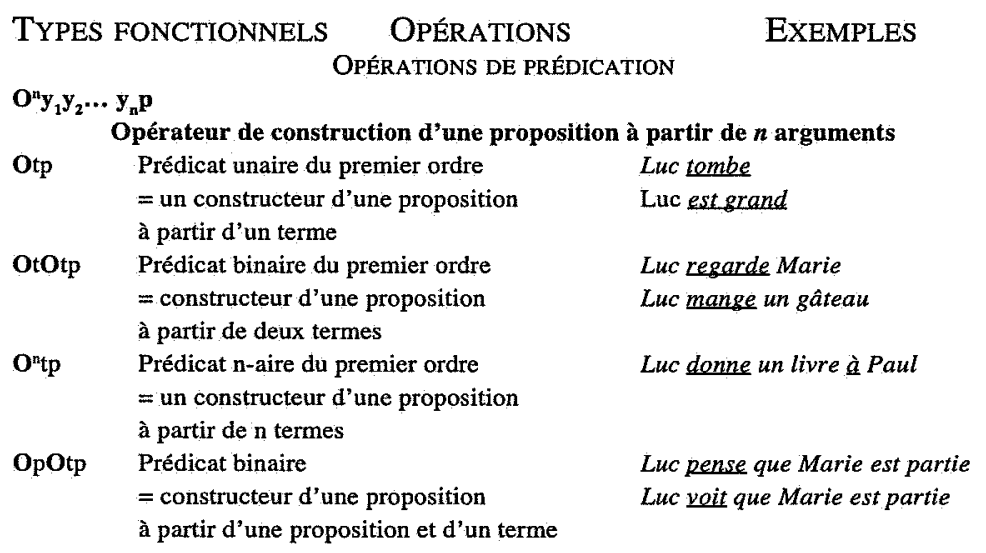




Oxx
Ott
OOtpOtp
OO'tpOntp
Opp

OxOxx

Otott

OpOpp

OOtpOOtpOtp

OyOxx

OtOOtpOtp

Oyt

OOtpt

$\mathbf{O O}^{\mathrm{t}} \mathrm{pt}$

OyOtp

OtOtp

OOttOtp

OOOtpOtpOtp

OOtp (Otp) $)^{\mathrm{n} p}$

$(\mathrm{n}=\mathbf{0}, \mathbf{1}, \mathbf{2 .}$.)

OOtpp

OOtpOOtpp

OOtpOO $^{n}$ tpp
OPERATIONS DE DETERMINATION

Opérateurs de détermination d'une unité de type $x$

Déterminant $\mathrm{d}^{\prime}$ un terme Un enfant joyeux

Déterminant d'un prédicat unaire Luc court vite

Déterminant d'un prédicat $\mathbf{n}$-aire

Opérateur de modalité il estpossible qu'il vienne

= détermine une proposition cen'est pas le cas qu'il vienne

OPERATIONS DE CONNEXION

Connecter deux unités de type $x$

Connecteur de deux termes Pierre quec Marie

Connecteur de deux propositions Il pleut et il fait du vent

Connecteur de deux Prédicats Il est bête et est méchant

OPERATIONS DE TRANSPOSITION

Transposition vers un opérateur de détermination

Transposer un terme en un adverbial

dans la chambre

Transposer un terme en un circonstanciel

dans l'après-midi

Transposition vers un terme

Transposer un prédicat unaire

en un terme construction d'un terme à

partir d'un prédicat

\author{
nag-eur \\ construc-tion \\ est un homme \\ est mortel \\ est dessous
}

Construction d'un prédicat à partir

d'un déterminant adverbial

Opérations de quantification frégéenne

Quantification sur un prédicat unaire

$\rightarrow$ transposer un prédicat en une

tous sont-mortels

proposition

Quantification sur un prédicat unaire,

restreinte par un prédicat

Quantification sur un prédicat n-aire,

restreinte par un prédicat

tout homme donne

\section{Convergences et divergences}

Cette approche de l'analyse linguistique par différents types fonctionnels d'opérateurs systématise, plus ou moins directement, un certain nombre de conceptualisations opérées par certains modèles. On reconnait facilement les liens avec la 
théorie de la valence de l'analyse par les stemmas de L. Tesnière (1966), sous la forme d'opérateurs (verbaux), c'est-àdire en fait des prédicats lexicaux, qui sont appliqués à des opérandes (les actants) pour construire des phrases ${ }^{17}$. Il y a cependant quelques différences entre d'un côté, les «arbres applicatifs » construits par l'application des opérateurs aux opérandes adéquats et d'un autre côté, les stemmas (de dépendance $)^{18}$. La première différence tient à une hiérarchisation des arguments d'un prédicat, effectuée par l'opération globale qui opère en enchainant les opérations élémentaires de prédication. En effet, dans un stemma de Tesnière, les différents actants sont situés au même niveau de dépendance par rapport au prédicat (le verbe) ${ }^{19}$ alors que dans un arbre applicatif, l'opération de prédication s'effectue en plusieurs temps, établissant ainsi une hiérarchisation entre les différents opérandes (actants ou arguments) qui entrent dans la structure prédicative. Ainsi, le sujet syntaxique, appelé «terme primaire» par S.K. Shaumyan (1987], se définit comme étant «le dernier opérande qui entre dans la construction prédicative d'une phrase $^{20} \gg$. L'opération de prédication, en hiérarchi-

17. Les opérations de transposition formalisent exactement les opérations de translation de Tesnière.

18. Plus généralement, les «arbres de dépendance» (par exemple Mel'čuk, 1987) introduisent des relations entre un opérateur et un argument.

19. C'est le cas également de la logique classique (du premier ordre) qui met au même niveau hiérarchique les différents arguments du prédicat.

20. Cette approche s'oppose à la théorie plus classique des compléments (par exemple chez O. Jespersen (1971), dans laquelle le «sujet syntaxique "serait un complément de «rang zéro» ou «complément obligatoire», les autres compléments étant introduits ensuite dans la relation prédicative. Ainsi, dans l'approche par complémentation, le prédicat s'applique d'abord au sujet puis ensuite au complément d'objet direct, puis aux autres compléments... Plusieurs arguments (morphologiques, syntaxiques, sémantiques, logiques, psychologiques sur l'acquisition et la mémorisation des structures verbales...), que nous ne donnons pas ici, faute de place, nous amènent à considérer que c'est le sujet syntaxique qui est le dernier argument qui entre dans la construction de la relation prédicative, et non pas le premier comme dans la plupart des analyses formelles. Le formalisme des types fonctionnels et des 
sant l'entrée des arguments du prédicat dans la construction prédicative, est étroitement liée au "principe de curryfication » qui associe canoniquement à tout prédicat $n$-aire ' $P_{n}$ ', de type ' $O^{n}$ tp', un opérateur unaire ' $Q_{1}$ ', de type 'Ot $O^{n-1}$ tp ; en s'appliquant à un terme $T, l^{\prime}$ 'opérateur ' $Q_{1}$ ' construit un prédicat (n-1)-aire, qui à son tour s'appliquera à un autre terme. Ainsi on peut comparer les deux structures prédicatives suivantes $^{21}$ :

$P_{n}\left(<T^{1}, T^{2}, \ldots, T^{n-1}, T^{n}>\right) \quad$ et $\quad\left(\ldots\left(\left(P_{n} T^{n}\right) T^{n-1}\right) \ldots T^{2}\right) T^{1}$

La première structure ${ }^{22}$ résulte d'une opération globale de prédication qui applique directement le prédicat $\mathrm{P}_{\mathrm{n}}$ à un $\mathrm{n}$ uple $\left.\left(<T^{1}, T^{2}, \ldots, T^{n-1}, T^{n}\right\rangle\right)$, elle $n^{\prime}$ implique ainsi aucune hiérarchie entre les arguments; la seconde structure résulte d'une opération de prédication qui établit une hiérarchie entre les termes qui entrent successivement dans la construction prédicative. La hiérarchisation des termes dans une construction prédicative est pertinente en linguistique, elle permet par exemple d'analyser les différences casuelles dans une langue à cas, les différences de position syntagmatique, les différences d'accord (en français, le verbe s'accorde avec le sujet syntaxique).

opérateurs est cependant neutre par rapport à ces deux positions théoriques puisqu'il permet de les exprimer formellement toutes les deux. C'est donc une argumentation linguistique qui doit choisir une voie et rejeter l'autre. La formalisation, avec un formalisme riche et expressif, comme celui des opérateurs typés, offre justement aux linguistes l'obligation de choisir entre deux organisations a priori possibles, et ainsi de discuter explicitement une décision linguistique, justifiée dans un cadre théorique précis, que le formalisme peut, par ailleurs, exprimer adéquatement. Ainsi, le formalisme propose des solutions formelles mais seule une argumentation linguistique peut choisir une solution acceptable.

21. Le «principe de curryfication» (Desclés, 1990; Hindley et Seldin, 1986) revient à exprimer la correspondance bijective entre les deux types ' $O^{2} y x$ 'et 'OyOyx'. Cette bijection est parfaitement justifiée dans la catégorie des ensembles puisque, en désignant par 'Ens $(\mathrm{Y}, \mathrm{X})$ ' $l$ 'ensemble de toutes les applications de l'ensemble ' $Y$ 'dans l'ensemble ' $X$ ', nous avons la bijection entre les ensembles d'application 'Ens $\left(\mathrm{Y}_{1} \mathrm{XY}_{2}, \mathrm{X}\right)$ ' et 'Ens $\left(\mathrm{Y}_{2}\right.$, Ens $\left(\mathrm{Y}_{1}, \mathrm{X}\right)$ ' et 'Ens $\left(\mathrm{Y}_{1}\right.$, Ens $\left(\mathrm{Y}_{2}, \mathrm{X}\right)$ '.

22. Cette structure est celle qui a été retenue pour l'analyse de la prédication dans les langages logiques du premier ordre, qui ne hiérarchise pas les arguments d'un prédicat. 
La seconde différence touche à la détermination. L'opération de détermination est essentielle dans l'organisation des agencements linguistiques des langues ${ }^{23}$. Nous y reviendrons plus loin. Dans l'opération de détermination, le déterminant (par exemple un adjectif ou un adverbe ou encore une relative) de type 'Oxx' est un opérateur qui a pour opérande l'élément déterminé, apportant à ce dernier une information supplémentaire, qualificative et souvent restrictive. L'arbre applicatif qui représente cette construction met l'opérateur de détermination «au-dessus de» son opérande, le résultat obtenu constituant une expression du même type que celui de l'opérande: le déterminant est un opérateur qui s'applique à une opérande qui ainsi devient mieux déterminé. Dans les arbres de dépendance et dans les stemmas de Tesnière, le déterminant (par exemple un adjectif ou un adverbe) « dépend de» l'élément déterminé (un nom dans le cas de l'adjectif; un verbe dans le cas de l'adverbe). Ainsi, les structures des arbres ne sont plus exactement isomorphes: dans les arbres applicatifs, l'opérateur de détermination «domine » l'élément déterminé - son opérande - tandis que dans les arbres de dépendance, l'élément (devant être) déterminé est «le père» de l'élément déterminé, qui ainsi en dépend en tant qu'expansion (Desclés, 2000).

La notion de «tête» - thématisée dans les modèles comme HPSG -, avec les «expansions » qui en dépendent, est analysée aussi bien par les arbres de dépendance que par les arbres applicatifs. En effet, dans ces derniers, la «tête» d'un syntagme construit avec ses expansions (en fait des déterminations successives), est le premier opérande de type ' $x$ 'sur lequel s'appliquent successivement des opérateurs de détermination, de type 'Oxx'; 1 'accord déterminant-déterminé (par exemple en genre et en nombre), quand il est exprimé, est

23. La logique classique depuis G. Frege ignore les opérations de détermination, en ramenant ces dernières à des prédications. Ainsi, un nom commun, un verbe intransitif, un adjectif sont considérés, en logique classique, comme des prédicats. La logique ante-frégéenne, par exemple la Logique de Port Royal, tenait compte d'opérations de détermination, sans pour autant les avoir formalisées. 
imposé par l'opérande ( $«$ la tête $\left.{ }^{24}\right)$. La structure applicative permet de formaliser complètement la notion de dépendance en particulier celle de "tête » avec ses expansions - en indiquant explicitement, par le type 'Oxx', que l'opérateur de détermination (un déterminant) «dépend de son opérande» (le déterminé) puisqu'il faut avoir déjà construit l'élément à déterminer avant de pouvoir y appliquer l'opérateur de détermination. La représentation applicative introduit cependant des informations supplémentaires qui sont exprimées par une hiérarchisation, exprimée par un ordre applicatif, que l'analyse sémantique ultérieure peut exploiter directement. En effet, du point de vue référentiel, chaque détermination restreint, ou élargit (avec par exemple les opérateurs de quantification), «l'extensité» (au sens de Wilmet, 1986) d'un terme nominal et modifie l'extension d'un prédicat verbal. Chaque détermination, par exemple à l'aide d'une préposition, spécifie l'opérande sur laquelle porte cette opération.

La notion d'opérateur et d'opérande se retrouve, avec de nombreuses nuances cependant, dans d'autres approches théoriques comme 1'apport (opérateur) et le support (opérande) chez G. Guillaume (1964) et chez B. Pottier (1974, 1992,2000 ) et également, chez M. Le Guern (2003) ou chez A. Berrendonner (1981). Outre dans la Grammaire applicative de S.Shaumyan (1965, 1987), Desclés (1990), l'opération d'application d'un opérateur à un opérande est également présente dans la théorie de A.Culioli (Culioli et al., 1970; Culioli, 1990, 2002) (voir aussi Desclés, 1992, 1995, 1999, 2006).

Lorsqu'on examine la théorie des opérateurs de la théorie de Z. Harris $(1968,1976,1982,1988)$, on retrouve les différents types fonctionnels, qui sont des analogues des types fonctionnels des Grammaires catégorielles ${ }^{25}$. En effet,

24. La notion d'accord peut être traitée dans les grammaires catégorielles au moyen d'opérateurs métalinguistiques que nous ne présentons pas ici,

25. «Il faudrait noter que la relation opérateur-argument produite par cette dépendance présente des similarités importantes avec les foncteurs de la grammaire catégorielle en logique». (Harris ; 2007 : 37). L'orientation des types est implicite chez Harris. 
Z. Harris utilise différents types d'opérateurs. Sans entrer dans les détails et en prenant pour types de base les types ' $n$ '. (des entités nominales) et o'(des discours), nous retrouvons facilement les liens avec la théorie de la valence de Tesnière, c'est-à-dire avec les opérateurs dont les types sont respectivement ${ }^{26}$ 'Ono', ' $\mathbf{O}^{2}$ no', ' $\mathbf{O}^{3}$ no'. D'autres opérateurs sont introduits par Z. Harris $(1976,1982)$, par exemple: 'OoOno' ( $e$ sais qu'il est ici); 'OnOoo'(que Jean traverse la rue m'a surpris), 'OnOnOoo'(Jean a demandé à Frank si Marie avait gagné le prix); 'OoOoo'(Que Jean ait téléphoné a entrainé le retour de Frank). Harris utilise également des «opérateurs de méta-discours» de type 'Ooo'nécessaires à l'identification d'adresses positionnelles, exprimée par exemple:

le second argument du premier argument du discours opérande est identique au second argument du second argument du discours opérande.

Cet opérateur de méta-discours est appliqué au discours opérande suivant:

$J$ 'ai acheté un livre et elle a acheté un livre

en construisant alors le résultat:

J'ai acheté un livre et elle en a acheté un.

Dans la théorie de Z. Harris, les opérateurs de différents types et les opérateurs de méta-discours sont organisés pour construire des expressions complexes (et directement interprétables) qui sont ensuite «réduites», par différents opérateurs transformationnels, formalisés par des opérateurs de composition $^{27}$, afin d'obtenir des expressions applicatives directement associées aux structures de surface des discours.

26. Les notations utilisées par Harris (1976) sont respectivement pour tous les opérateurs que nous allons évoquer dans ce paragraphe: 'On', 'Onn', 'Onnn', 'Ono', 'Oon', 'Onno', ‘Ooo', 'Oo'...

27. Il nous parait possible de montrer explicitement que les opérateurs transformationnels qui permettent les réductions dans la théorie de Z. Harris sont entièrement formalisables à l'aide des combinateurs de la logique combinatoire de H. Curry et R. Feys (1958). La preuve effective reste encore à faire. 


\section{Langage logique du premier ordre}

Pour mieux discuter des approches logiques et linguistiques du langage et des langues, il est intéressant de bien spécifier les types des «constantes logiques », c'est-à-dire des opérateurs logiques du langage des propositions (connecteurs logiques) et du langage des prédicats (quantificateurs frégéens). Un «langage logique du premier ordre ${ }^{28} »$, désigné désormais par LPO, est constitué d'unités, données initialement ou construites au moyen d'opérateurs; ces unités sont d'un côté, les termes et les propositions du langage, et d'un autre côté, les constantes logiques exprimées par des opérateurs de différents types:

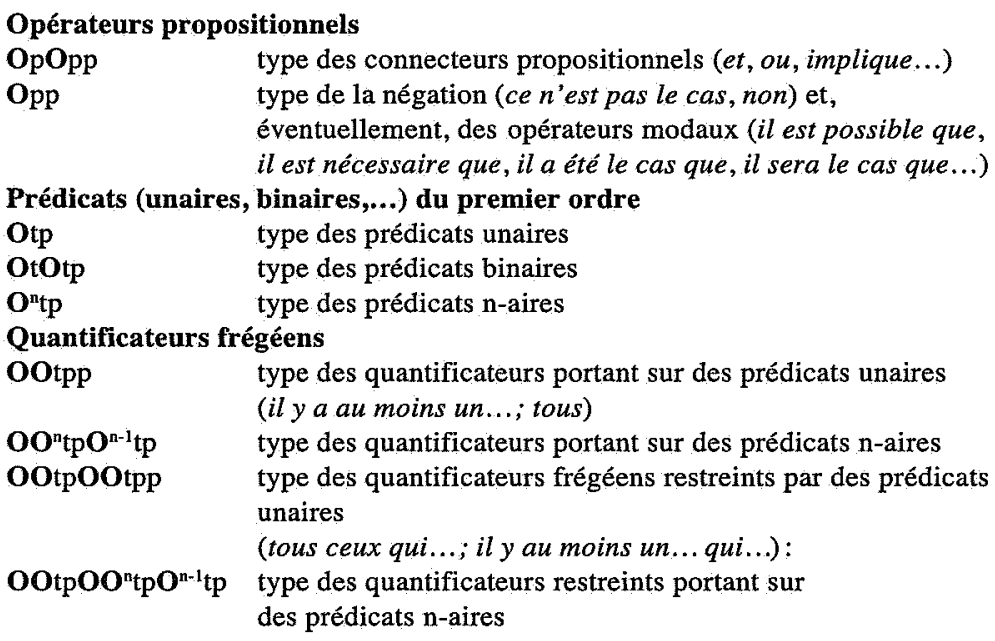

On peut y adjoindre, éventuellement, dans un LPO, des opérateurs de type 'OOtpt' (des constructeurs de termes à partir de prédicats) comme l'opérateur de sélection d'un terme indéterminé (un qui est...) représentant, en tant que terme, un prédicat unaire (c'est le -symbole de Hilbert ou l'opérateur de Bourbaki ${ }^{29}$ ) et l'opérateur «iota» de la description définie proposé par B. Russell (le qui est...).

28. Il s'agit du «Calcul des prédicats» classique, présenté par exemple par W.V.O. Quine (1970).

29. Dans le cadre de la Logique de la Détermination des Objets (LDO) (Desclés et Pascu, 2006; Pascu, 2006), nous interprétons 


\section{Prédication seconde}

On rencontre parfois dans la littérature linguistique l'expression de «prédication seconde» sans que celle-ci ait reçu une définition formelle très claire. Dans certains cas, il est correct de considérer que certaines unités linguistiques fonctionnent bien comme des prédicats mais, dans d'autres cas, 1'appellation de «prédicat second» nous semble pouvoir entrainer des confusions regrettables qui amèneraient vite à interdire toute confrontation fructueuse entre analyse linguistique et analyse logique. Ces deux analyses, bien que différentes parce que les buts poursuivis ne sont pas identiques, ne sont pas indépendantes et doivent au contraire s'articuler étroitement, l'histoire montrant constamment, l'intrication entre grammaire et logique depuis l'origine de ces disciplines. Lorsqu'un prédicat comme voit ou pense est constitutif d'une proposition, il prend pour l'un de ses arguments une proposition déjà constituée; dans de tels cas, son type est 'OpOtp'. La construction applicative de la proposition Paul pense que Marie est déjà venue est réalisée comme suit:

déjà và venue
déjà venue
est
(est (déjà venue)) Marie
pense ((que ((est (déjà vènue venue)) Marie))

où les types assignés sont respectivement: [Marie: $\mathrm{t}] ;[$ Paul: $\mathrm{t}] ;$ [venue: Ott] ; [déjà: OOttOtt]; [est: OOttOtp] ; [que: Opp]; [pense: OpOtp] ${ }^{30}$. Considérer pense ou pense que

l'opérateur $\tau$ comme un constructeur d'un «objet typique complètement indéterminé».

30. De façon plus exacte, que est un opérateur dont le type et 
comme des prédicats, appelés des «prédicats seconds», est tout à fait acceptable Cependant, dans d'autres cas, comme :

(a) Manifestement, Marie n'a rien compris

(b) Malade, Marie est restée chez elle

il nous parait peut-être abusif de considérer que manifestement et malade sont des prédicats seconds. En effet ces deux unités linguistiques fonctionnent bien comme des opérateurs qui ont pour opérandes des propositions déjà constituées. Dans une analyse superficielle, ces opérateurs pourraient se voir assigner les types respectifs 'Opp' (comme modificateur adverbial de la proposition), Ott (comme modificateur adjectival du nom). Cependant de telles analyses sont insuffisantes puisqu'elles ne traduisent pas exactement les interprétations sémantiques des phrases en question. Ces dernières sont les résultats d'opérateurs transformationnels (au sens de Z. Harris) qui réduisent des formes plus complexes sous-jacentes à des expressions plus compactes. La première phrase (a) Manifestement, Marie n'a rien compris est la réduction paraphrastique d'une forme plus élaborée (a'), la seconde (b) Malade, Marie est restée chez elle est la réduction d'une relation (b')

(a') Marie n'a rien compris est un fait manifeste

(b') Marie est malade est la cause de Marie est restée chez elle

Dans ces formes (a') et ( $\left.b^{\prime}\right)^{31}$ sous-jacentes à (a) et à (b), les opérateurs est un fait et est la cause de sont des opérateurs métalinguistiques qui ont bien le statut de prédicat, le premier a de type 'Opp', le second a le type 'OpOpp'. Ces deux opé-

1'analyse sont complexes. En lui assignant le type Opt, il est composable avec 1'opérateur pense (de type OtOtp), il en résulte 1'opérateur complexe pense que de type 'OpOtp'. Pour réaliser cette composition fonctionnelle d'opérateurs, il nous faut introduire les opérateurs de composition que sont les combinateurs de la logique combinatoire (Curry et Feys, 1958). Pour l'utilisation des combinateurs dans l'analyse du langage, voir Shaumyan (1987) et Desclés (1990).

31. Ces formes sous-jacentes sont des représentations indispensables aux interprétations. Les formes superficielles donnent seulement certaines instructions pour construire des formes interprétatives plus élaborées avec l'introduction d'opérateurs métalinguistiques. 
rateurs sont des constructeurs de propositions à partir d'une (respectivement deux) proposition(s). Ils ont donc une fonction prédicative: construire une proposition. Il apparait bien par cette analyse que les opérateurs manifestement et malade, en position détachée, ne fonctionnent alors pas comme des prédicats (même qualifiés de «seconds») mais comme étant issus de la réduction de formes construites avec des prédicats métalinguistiques portant sur une ou plusieurs propositions ${ }^{32}$. Dans le même ordre d'idées, la phrase

(c) Marie a les yeux verts

pourrait être analysée par une expression superficielle de la forme applicative préfixée :

(c') (a (les (verts yeux) Marie

Dans (c'), verts est un adjectif (de type Ott) qui vient déterminer le terme nominal yeux avant que la détermination complète (donc susceptible de construire un objet référentiel) soit réalisée par l'article les; l'opérateur $a$ (de type OtOtp) met en relation deux nominaux en s'appliquant, dans un premier temps, au terme les yeux verts construisant ainsi un Prédicat qui, dans un second temps, s'applique à Marie. Cependant, certains auteurs analysent cette phrase au moyen du «prédicat second" les yeux verts qui construirait, selon eux, une prédication seconde sous la forme d'une proposition attributive emboitée. En admettant cette analyse plus complexe et en nous situant dans la théorie des opérateurs de Z. Harris, nous considérons que la forme sous-jacente (c') de (c) serait obtenue en coordonnant deux propositions plus élémentaires liées par une pronominalisation:

(c") Marie a des yeux et ses yeux sont verts

Dans ce cas, l'opérateur les yeux verts serait issu d'une réduction de la forme (c') qui fait intervenir (i) le connecteur et; (ii) un opérateur de méta-discours d'identification dont la trace linguistique est ses; (iii) le Prédicat sont verts construit

32. L'utilisation des combinateurs de la logique combinatoire devient nécessaire pour préciser jusque dans le détail les opérations transformationnelles de réduction, en particulier les compositions d'opérateurs, les déplacements et permutations d'arguments et les effacements (Desclés, 1990). 
à partir de l'adjectif vert au moyen du constructeur est. Dans ce cas, il nous semble extrêmement dangereux de faire appel à une prédication seconde car la forme sous-jacente et le mécanisme de réduction impliquent des opérations métalinguistiques plus complexes et de nature différente que la simple opération de prédication exprimée dans ( $\left.c^{\prime}\right)$.

\section{Une analyse non frégéenne des quantificateurs}

Comme nous l'avons déjà dit, le langage logique du premier ordre (LPO) ne possède aucun opérateur de détermination. Ainsi, lorsqu'on analyse les langues naturelles à l'aide d'un LPO, les opérations de détermination sont «ramenées» à de simples opérations élémentaires de prédication. Ainsi, la phrase (a), lorsqu'elle est analysée dans le LPO, est représentée, à l'aide de variables liées, par la proposition (b), dont (c) serait la paraphrase directe ${ }^{33}$ :

33. Cette analyse s'inscrit dans la tradition, revendiquée par W.O. Quine (1970), de la «forme logique» sous-jacente à une phrase. La notion de «variable liée» apparait comme étant nécessaire dans la plupart des «présentations» sémiotiques de la Logique du Premier Ordre (LPO), en particulier dans la présentation qu'en font B. Russell et O.W. Quine, et, à leur suite, la plupart des manuels de logique. Le recours aux variables liées a cependant des inconvénients : il rend peu transparent le rôle fonctionnel des quantificateurs (Desclés et Cheong, 2006). D'autres présentations sémiotiques des LPO font l'économie des «variables liées », c'est le cas de la présentation retenue par H. Curry et R. Feys (1958) avec la «logique illative» qui ne fait plus appel aux variables liées dans la présentation des quantifícateurs (formalisant en fait sans variables liées l'appareil issu de G. Frege $(1893,1971)$. Cette dernière est replacée dans le cadre plus général de la logique combinatoire, considérée alors comme une «prélogique». On remarquera, par ailleurs, que le v-calcul de Church (1941) (voir Hindley et Seldin (1986), Desclés $(1990,2004)$ fait largement appel à des «liaisons entre des occurrences d'une même variable " pour exprimer l'action d'opérateurs, là où la logique combinatoire utilise des combinateurs dont les actions sont alors spécifiées par des règles d'introduction et d'élimination, dans le style de Gentzen, (Fitch, 1974 ; Desclés, 1990). 
(a) Tout homme honnête est admirable

(b) $(\forall x)$ [est-homme $(x) \wedge$ est-honnête $(x) \rightarrow$ estadmirable (x)]

(c) Pour tout individu, s'il est un homme qui est honnête alors il est admirable

Dans cette analyse, on voit que l'adjectif honnête est représenté par le Prédicat «est-honnête», que le terme nominal homme est représenté par le Prédicat «est-homme»; ainsi, la distinction morphologique du français, et dans de nombreuses langues, entre nom commun, adjectif et prédicat verbal n'est pas conservée, bien que du point de vue sémantique, on puisse trouver plusieurs critères pour les distinguer ${ }^{34}$. La représentation (b) ne permet pas de refléter les distinctions catégorielles opérées par le français; de plus, elle introduit un artefact représentationnel sous la forme d'occurrences de la variable ' $\mathrm{x}$ ', liées par le quantificateur universel, conduisant alors à des paraphrases très lourdes et souvent peu naturelles ${ }^{35}$. Pour notre part, nous considérons que 1'analyse applicative (d) de l'énoncé (a) respecte beaucoup mieux les distinctions catégo-

34. Donnons quelques arguments sémantiques. Par exemple, un adjectif exprime souvent une propriété stative, alors que certains verbes intransitifs, comme tombe, expriment plutôt des actions (donc des propriétés aspectuelles de «processus»). Un adjectif ajoute une propriété déterminative à un terme nominal déjà constitué, modifiant ainsi son extensité (Wilmet, 1986) ou son «étendue », pour reprendre la Logique de Port Royal. On peut considérer qu'un terme nominal comme un homme, désignant un objet individuel complètement indéterminé, a été construit à partir du concept «être-homme» par une opération explicite (l'opérateur $\tau$ dans la Logique de la Détermination des Objets - LDO); plusieurs déterminations peuvent venir ensuite s'ajouter à ce terme indéterminé pour préciser en partie sa référence, mais la plupart des adjectifs ne possèdent pas cette propriété puisqu'ils nécessitent des supports (dénotant des objets) pour pouvoir s'appliquer : si un homme désigne un objet indéterminé, un rouge ne désigne rien (sauf dans certains contextes avec renvois anaphoriques).

35. Pour certains auteurs, comme O.W. Quine, la «variable liée» serait la formalisation du jeu des substituts anaphoriques exprimés par les pronoms des langues naturelles. Cette position peut être fortement discutée car, dans les paraphrases interprétatives de (b), il faut introduire les opérateurs de métadiscours de Z. Harris $(1976,1982)$ pour identifier les adresses des occurrences. 
rielles du français mais en traitant le quantificateur le syntagme quantifié tout honnête homme comme un opérateur portant sur le Prédicat est admirable ${ }^{36}$ :

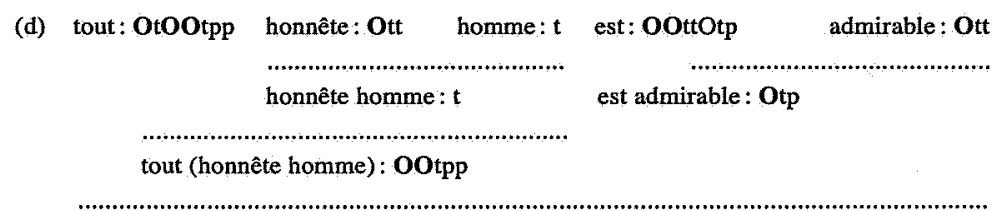

(tout (honnête homme) (est admirable): p

Comme nous l'avons argumenté dans d'autres articles (Desclés \& Guentcheva, 2001 ; Desclés, 1999, 2005), une représentation métalinguistique adéquate de (a) n'est ni (b), ni même (d) mais plutôt la représentation (e) (présentée selon un ordre applicatif préfixé) $)^{37}$ :

(e) (est admirable) (tout (honnête homme)

qui n'analyse pas, maintenant, le syntagme quantifié tout homme honnête comme un opérateur mais comme un terme nominal. Cette analyse applicative reste ainsi directement compatible avec l'analyse syntaxique, par exemple celle très classique en constituants immédiats; elle ne transforme pas, comme le fait R. Montague et, à sa suite un certain nombre de linguistes, une représentation syntaxique, dans la représentation logique (b), en voulant rester ainsi conforme à l'analyse frégéenne de la quantification. L'analyse exprimée par la représentation (e) nécessite, en revanche, l'introduction de «quantificateurs non frégéens » plus adéquats, selon nous, à 1'analyse de la quantification et de la prédication opérées par les langues naturelles que celle qui est héritée de Frege. En effet, par cette représentation (e), l'analyse du syntagme

36. Ce syntagme nominal est alors substituable à n'importe quel «nom propre», argument du Prédicat. Cette analyse est, sur le plan intuitif, plus conforme aux analyses logiques ante-frégéeennes (Logique de Port Royal par exemple) qui cherchaient à rester plus proches des structures linguistiques que l'actuelle logique.

37. On peut comparer (e) '(est admirable) (tout (honnête (homme)' et la représentation (d') construite par (d): (d') '(tout (honnête (homme) (est admirable)' ; la première met le syntagme quantifié en position d'argument, la seconde met le syntagme quantifié en position d'opérateur. 
quantifié tout homme honnête reste argument du Prédicat (logique) est admirable, et fonctionne ainsi comme son Sujet (logique), ce que l'analyse syntaxique de (a), effectuée dans le cadre des Grammaires Catégorielles, exprimerait par la construction (f), que le quantificateur tout fonctionne comme un déterminant ${ }^{38}$ qui peut spécifier ainsi, du point de vue d'une sémantique référentielle, «l'extensité» du syntagme nominal :

(f)

\begin{tabular}{|c|c|c|c|c|}
\hline $\begin{array}{l}\text { Tout } \\
\mathrm{N}^{*} / \mathrm{N}\end{array}$ & $\begin{array}{l}\text { homme } \\
\mathrm{N}\end{array}$ & $\begin{array}{l}\text { honnête } \\
\text { NWN }\end{array}$ & $\begin{array}{c}e s t \\
(\mathrm{SWN}) /(\mathrm{N} N \mathrm{~N})\end{array}$ & $\begin{array}{l}\text { admirable } \\
\mathrm{NLN}\end{array}$ \\
\hline & & $\mathbf{N}$ & & \\
\hline
\end{tabular}

Cette analyse, et la représentation applicative (e) qui en résulte, maintient un parallélisme étroit entre d'une part, les analyses des constructions prédicatives avec syntagmes quantifiés, comme dans tout homme vertueux est admirable ou dans il y a au moins un homme vertueux qui est admirable et d'autre part, les analyses des mêmes constructions prédicatives avec syntagmes non quantifiés, comme Paul est admirable ou le petit garçon que tu connais bien est admirable. L'introduction de quantificateurs non frégéens, sous la forme $\mathrm{d}^{\prime}$ 'opérateurs de détermination ${ }^{39}$, permet de relier ces derniers

38. Dans une analyse catégorielle qui souhaite construire des représentations proches des représentations logiques obtenues avec les langages du premier ordre, le quantificateur restreint tout se voit attribuer, comme dans les analyses de R. Montague (1974), le type syntaxique ' $\left(\mathrm{S} /\left(\mathrm{S} \mathrm{N}^{*}\right) / \mathrm{N}^{*}\right.$ ', ce qui conduit alors à une représentation prédicative où le syntagme quantifié tout homme honnête fonctionne comme un opérateur dont le Prédicat est admirable est devenu un opérande.

39. Les quantificateurs frégéens classiques sont reliés (sans leur être équivalents) aux quantificateurs non frégéens que nous avons introduits. Pour établir cela, il faut se placer dans le cadre de la logique combinatoire de Curry et Feys (1958); Desclés $(1990,2004$ b, 2005). Les quantificateurs non frégéens (dits «stars») apparaissent alors comme plus primitifs que les quantificateurs frégéens qui deviennent dérivés. Pour 
à d'autres déterminants complexes comme les articles ${ }^{40}$, et également de mettre en correspondance (compositionnelle) les analyses syntaxiques et les représentations logico-grammaticales préparatoires aux interprétations sémantiques ultérieures.

\section{Analyse des rôles grammaticaux}

Les représentations prédicatives construites à la suite d'analyses catégorielles aboutissent à des représentations métalinguistiques conçues comme des agencements bien formés d'opérateurs appliqués à des opérandes de différents types en respectant toutes les contraintes imposées par les types. Ces représentations métalinguistiques bien formées mettent ainsi en évidence le rôle opératoire et combinatoire des différentes opérations constitutives des énoncés et phrases: prédication, détermination, quantification, transposition... Cependant, pour une analyse grammaticale des énoncés, il faut recourir, entre autres, aux rôles grammaticaux (appelés également, selon les théories, «relations casuelles», «relations thématiques» ou encore «cas conceptuels») comme «agent de», «patient de», «instrument de», «cause naturelle de», «origine de », «but de», «destinataire de», «localisateur», «lieu intermédiaire ${ }^{41} \gg \ldots$ Pour formaliser ces rôles, il faut donc enrichir les langages applicatifs d'opérateurs typés, c'est-à-

une critique de la quantification frégéenne appliquée aux représentations des langues naturelles, voir Sommers (1982); Bach et al. (1995); Desclés (1999, 2005); Desclés et Guentcheva (2001); Desclés et Cheong (2006); Desclés et Pascu (2006); l'analyse de la quantification de C.S. Peirce (Chauviré, 2006).

40. Le type syntaxique des articles et des quantificateurs (stars) est, dans notre approche, ' $N * / N$ ', signifiant par là que les articles et les quantificateurs nominaux sont des opérateurs, appelés parfois des actualisateurs, qui construisent des syntagmes nominaux complets, dont l'extensité peut être ainsi déterminée.

41. Les LPO ne permettent ni de représenter les relations casuelles ni de construire des prédicats gouvernant des cas. 
dire : 1) introduire des « constantes casuelles ${ }^{42} \gg$ sous la forme d'opérateurs spécifiques aux organisations des langues naturelles; 2) indiquer leurs modes de composition avec les prédicats et les autres opérateurs linguistiques (déterminants, connecteurs, transposeurs...); 3) préciser la sémantique interprétative de ces opérateurs. Plus généralement, il faut introduire, dans le formalisme applicatif typé, des opérateurs grammaticaux (par exemple opérateurs de diathèse, de thématisation...) spécifiques à l'analyse des langues (donc non logiques) pour décrire les opérations portant sur les constructions prédicatives simples. Nous proposons une famille construite avec le même lexème verbal/sécher/, où apparaissent dans les différents énoncés divers rôles grammaticaux des sujets syntaxiques et différentes propriétés aspectuelles :

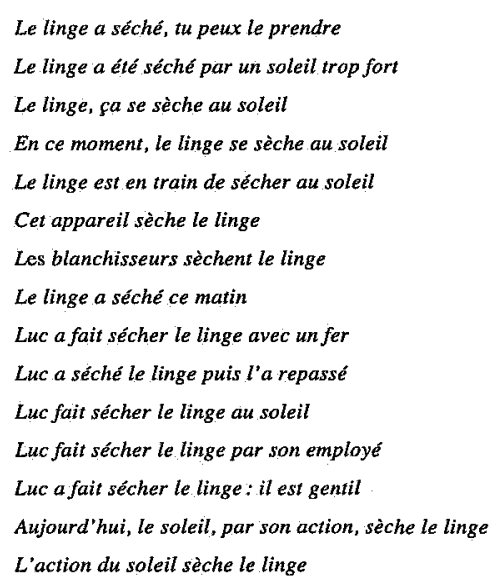

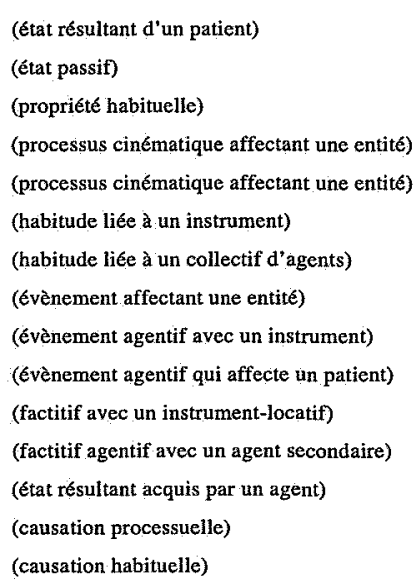

(causation habituelle)

42. Ces «constantes logiques» sont introduites sous la forme d'opérateurs spécifiques aux buts de la logique: étudier les inférences correctes et détecter les inférences incorrectes. Parmi les «constantes logiques », on met les opérateurs de négation, les connecteurs logiques (et, ou, si... alors...), les quantificateurs frégéens (tous, il y a au moins un...), les différents opérateurs modaux... dont on précise la sémantique, soit par des règles explicites (d'élimination ou d'introduction dans la déduction naturelle), soit par des jeux d'axiomes et des règles de dérivation, soit encore par des modèles ensemblistes d'interprétation. 
Pour illustrer très brièvement notre démarche ${ }^{43}$, nous allons prendre l'exemple de la relation d'agentivité, liée selon nous, essentiellement à la notion de «contrôle»: est agentive une entité «qui opère un contrôle, c'est-à-dire qui exerce le déclenchement ou l'interruption d'un changement, d'un mouvement, d'une transformation de propriétés... ${ }^{44}{ }$. Comment représenter la relation d'agentivité ? Comment se compose-telle avec les autres opérateurs? Pour y répondre rapidement, prenons les deux énoncés :

(a) Luc sèche le linge

(b) Le linge sèche

La phrase (a) exprime: (a') «un agent - Luc - exerce un contrôle sur une action qui affecte une entité - le linge dans »; (b) exprime (b') «une entité - le linge -, en tant que patient, est affectée par une transformation de ses propriétés passage d'un plus faible degré à un degré plus élevé de sécheresse - $\gg$. Nous représentons le rôle grammatical de «contrôle ${ }^{45} »$ dans (a) par le relateur 'CONTRÔLE' qui relie

43. Pour plus de détails, voir par exemple Desclés (1990, 2004 b, 2006).

44. La notion de «contrôle», liée à l'agentivité, a été introduite par Givòn; elle est utilisée par C. Hagège (Desclés, 1990). Remarquons que la notion de «contrôle» n'est pas ontologique car le contrôle, donc l'agentivité, se réalise à l'intérieur d'un discours. Certes, certaines entités sont des prototypes d'entités susceptibles d'exercer un contrôle. Nous avons donc une échelle d'agentivité depuis les humains, les animés... (Desclés, 1990, 1998, 2001, 2003); cependant, dans un contexte déterminé, une entité peut perdre le contrôle qu'elle possède habituellement (il est tombé par hasard) ou une entité qui normalement n'a pas le contrôle peut acquérir le contrôle d'une situation dans un contexte, par exemple par héritage (la voiture n'a pas respecté le feu rouge, elle est responsable de l'accident) ou par construction d'univers narratifs (le loup parla ainsi...).

45. Nous utilisons le relateur de 'CONTRÔLE' et non celui de 'CAUSE'comme le proposent plusieurs auteurs, par exemple D. Dowty (1979) ou J. François (1989) - pour représenter formellement l'agentivité. En effet, la "cause » est une relation entre deux situations distinctes, l'une étant la cause, l'autre l'effet. En revanche, dans une relation prédicative transitive comme (a), ou sa représentation (e) construite par l'intermédiaire de la définition (d), nous avons une seule situation référentielle 
l'entité agentive, qui exerce une capacité à déclencher ou à interrompre une action, et une relation prédicative qui exprime un changement de propriétés. Aussi l'énoncé (a) estil représenté par l'expression métalinguistique (c) ou, avec une notation préfixée, par ( $\left.c^{\prime}\right)$ :

(c) Luc CONTRÔLE ((le linge) sèche $\left.e_{1}\right)$

(c') ConTrốle (sèche ${ }_{1}$ (le linge) Luc

Les expressions (c) et ( $\left(c^{\prime}\right)$ font apparaitre explicitement le rôle casuel de l'agent. Le relateur 'CONTRÔLE' (en fait un opérateur binaire de type 'OpOtp') se compose avec le prédicat unaire sèche ${ }_{1}$ (de type Otp), pour construire un nouveau prédicat binaire (transitif) sèche $e_{2}$. (de type OtOtp). La composition doit s'effectuer à l'aide d'un opérateur. Désignons par 'B' l'opérateur de composition ${ }^{46}$ de 1 'opérateur grammatical CONTRÔLE avec sèche $e_{1}$; nous obtenons avec la définition du prédicat binaire dérivé séche $e_{2}$ et la représentation métalinguistique (e) associée à (a) :

[sèche ${ }_{2}={ }_{\text {def }}$ B CONTRÔLE (sèche $\left.e_{1}\right)$ ]

(e) sèche ${ }_{2}$ (le linge) Luc.

Dans l'expression (e), le prédicat sèche ${ }_{2}$ intègre le relateur (casuel) de "contrôle»; ainsi, dans (e), le terme Luc fonctionne alors comme «un agent» tandis que le terme le linge fonctionne comme «un patient». La relation entre (c') et (e) constitue ce que $\mathrm{Z}$. Harris appelle une relation de réduction, (e) étant la construction prédicative réduite et issue de (c'). Ces différents opérateurs, composés avec les prédicats de base, permettent d'en dériver des «prédicats complexes qui gouvernent des relations casuelles », c'est-à-dire des prédicats avec des rôles grammaticaux assignés aux arguments dans la relation prédicative construite.

où l'agent est l'un des participants qui contribue à la construire, c'est-àdire un actant qui a, ici, un rôle d'acteur agentif.

46. L'opérateur ' $B$ ' est un combinateur de la logique combinatoire (Curry et Feys, 1958; Desclés, 1990). Il permet, ainsi que les autres combinateurs, de composer fonctionnellement entre eux des opérateurs, de façon intrinsèque, c'est-à-dire indépendamment des domaines d'interprétation. L'action interne de ' $B$ ' est donnée par la règle 


\section{Conclusion}

Le cadre des opérateurs de différents types fonctionnels appliqués à des opérandes est adéquat pour étudier l'analyse des opérations constitutives mises en jeu par les langues, en maintenant une relation avec les opérations de la logique, ce qui permet de mieux en étudier les propriétés communes et surtout les différences et variations. La prédication est l'une des opérations constitutives des constructions exprimées par les langues. Elle implique donc des opérateurs prédicatifs (prédicats lexicaux élémentaires ou dérivés, Prédicats construits). Ces prédicats, en se composant avec des opérateurs grammaticaux destinés à établir des relations casuelles abstraites, donnent naissance à des «prédicats qui gouvernent des relations casuelles» et imposent des rôles grammaticaux aux arguments. En tant qu'opération, la prédication doit être distinguée d'autres opérations, tout aussi fondamentales, comme la détermination, les transpositions, les quantifications... sans parler de la thématisation (constituant dans l'énoncé une organisation «thème - rhème» qui vient se superposer à l'organisation prédicative $)^{47}$. Certains opérateurs sont des prédicats lorsqu'ils contribuent à construire des propositions ou des relations prédicatives complètes (ou des Prédicats encore incomplets). Il est clair que d'autres opérateurs, directement liés à certaines unités linguistiques, ne sont pas des prédicats, par exemple les connecteurs, les opérateurs de transposition... D'autres opérateurs sont plus abstraits, par exemple les opérateurs énonciatifs (de prise en charge aspecto-temporelle, d'engagement assertif et de désengagement...) et se compo-

d'élimination: Bfgx $\rightarrow \mathrm{f}$ ( $\mathrm{gx}$ ). Dans Desclés (1990, 2001, 2004 a et b, 2006), nous donnons de nombreux exemples d'utilisation des combinateurs pour des analyses grammaticales et sémantiques des langues naturelles. Sur la notion de «composition intrinsèque», voir Desclés et Cheong (2006).

47. L'analyse des opérations de thématisation (structuration thèmerhème) impose l'utilisation de combinateurs; elle ne peut pas être effectuée à l'aide des seuls types fonctionnels. Il en est de même pour l'étude des diathèses (constructions moyennes, passives, impersonnelles, réflexives...). 
sent avec d'autres opérateurs, laissant alors des traces linguistiques observables à partir desquelles il est possible de les reconstruire par une démarche abductive (Desclés, 2006).

En étendant, sans fournir une argumentation très serrée, le statut de prédicat (assimilé alors au terme beaucoup plus générique d'opérateur) et en qualifiant par «prédicat» presque tous les opérateurs linguistiques, on risque de ne pas faire apparaitre nettement les différentes opérations linguistiques mises en cuvre dans les structures linguistiques (avec des hiérarchisations d'opérations) spécifiques des langues naturelles et ainsi de ne pas distinguer nettement celles qui sont étroitement liées au lexique de celles qui sont spécifiquement grammaticales ou impliquées par la formalisation de la prise en charge énonciative. On risque ainsi de s'interdire toute comparaison fructueuse entre les structures logiques (analysées à l'aide des seuls langages du premier ordre) et les structurations sémiotiques effectuées par les langues. Le langage des types fonctionnels et des opérateurs de différents types est un outil formel qui nous permet de discuter en profondeur les rapports entre d'une part, le langage appréhendé au travers des langues, et d'autre part, les articulations entre les analyses logiques des énoncés et les représentations cognitives impliquées par les langues, ces représentations cognitives faisant, par ailleurs, appel au même formalisme.

\section{RÉFÉRENCES BIBLIOGRAPHIQUES}

Bach, E., JelineK, H., Kratze, A et PARTeE, B. (1995) : Quantification in Natural Languages, Kluwer Academic Press, 1995.

BERRENDONNER, A. (1981): Les référents nominaux et la syntaxe de l'énoncé, Thèse de doctorat d'État, Université de Lyon 2.

BISKRI, I. et DeSClÉs, J.-P. (1997): Applicative and Combinatory Categorial Grammar (from Syntax to Functional Semantics), in Recent Advances in Natural Languages Processing, John Benjamins Publishing Company, 71-84.

BISKRI, I. et DESCLÉS, J.-P. (2006a) : Coordination and Applicative Categorial Type Logic, Artificial Intelligence Tools, Architectures, Languages, Algorithms, vol 15, n6, 1007-1019. 
BISKRI, I. et DESCLÉs, J.-P. (2006b) : Coordination de catégories différentes en français, Faits de langues, 28, Ophrys, 37-66.

Chauviré, Ch. (2006): La quantification chez C.S. Peirce, in P. Joray (éd.), La quantification dans la logique moderne, Paris, L'Harmattan, 73-96.

Church, A. (1940): A Formalization of the Simple Theory of Types, Journal of Symbolic Logic, 5, 56-68.

CHURCH, A. (1941): The Calculi of Lambda Conversion, Princeton University Press.

Culioli, A., Fuchs, C. et Pecheux, M. (1970) : Considérations théoriques à propos du traitement formel du langage, Documents de linguistique quantitative $\mathrm{n}^{\circ} 7$, Paris, Dunod.

Culioli, A. (1990): Pour une linguistique de l'énonciation, Opérations et Représentations, tome 1, Paris, Ophrys.

Culioli, A. (1992): La théorie d'Antoine Culioli, Ouvertures et Incidences, Paris, Ophrys.

Culiol, A. (2002): Variations sur la linguistique, Paris, Klincksieck.

CURRY, H. (1961) : Some Logical Aspects of Grammatical Structure, in Structure of Language and its Mathematical Aspects, American Mathematical Society, Providence, Rhode Island, 56-68.

CURRY, H. et FeYs, R. (1958): Combinatory Logic, Amsterdam, North-Holland Publishing Company.

Desclés, J.-P. (1976): Description de quelques opérations énonciatives, in J. David, R. Martin (éditeurs), Modèles logiques et niveaux d'analyse linguistique, Paris, Klincksieck, 213-242.

DESCLÉs, J.-P. (1990): Langages applicatifs, langues naturelles et cognition, Paris, Hermès.

Desclés, J.-P. (1992): Au sujet des catégories grammaticales, in A. Culioli, 203-212.

Desclés, J.-P. (1995) : Schéma de lexis, in J. Bouscaren, J.-J. Franckel, S. Robert, Langues et langage, Problèmes et raisonnement en linguistique, Mélanges offerts à Antoine Culioli, Paris, Presses universitaires de France, 57-71.

Desclés, J.-P. (1998): Transitivité syntaxique, transitivité sémantique, in A. Rousseau, 1998, 162-180.

Desclés, J.-P. (1999): De la notion aux opérations de quantification, Les opérations de détermination Quantification/Qualification, Paris, Ophrys, 13-44.

DEsCLÉs, J.-P. (2000): Introduction aux grammaires catégorielles, Cours de DEA «Mathématiques, logique et linguistique», Université de Paris-Sorbonne, $80 \mathrm{p}$. 
DESCLÉs, J.-P. (2001): Prépositions spatiales, relateurs et préverbes, Etudes cognitives 4, Warszawa, Académie des Sciences de Pologne, Institut de slavistique, 13-30.

DESCLESS, J.-P. (2003) : Représentations cognitives, schèmes prédicatifs et schèmes énonciatifs, in Aboubakar Ouattara (éd.), Parcours énonciatifs et parcours interprétatifs, Théorie et applications, Paris, Ophrys, 21-46.

DEsClÉs, J.-P. (2004a): Analyse syntaxique et cognitive des relations entre la préposition SUR et le préverbe SUR- en français, Études cognitives, 6, Warszawa, Académie des Sciences de Pologne, Institut de slavistique, 21-48.

DESCLÉs, J.-P. (2004b) : Combinatory Logic, Language, and Cognitive Representations, in P. Weingartner (ed.), Alternative Logics. Do Sciences Need Them?, Springer, 115-148.

DESCLÉs, J.-P. (2005) : Une analyse non frégéenne de la quantification, in P. Joray (éd.), La quantification dans la logique moderne, Paris, L'Harmattan, 263-312.

DEsCLÉs, J.-P. (2006) : Opérations métalinguistiques et traces linguistiques, in D. Ducard, Cl. Normand (eds), Antoine Culioli, Un homme dans le langage, Colloque de Cerisy, Paris, Ophrys, 4169.

Desclés, J.-P. et Cheong K.-S. (2006): Analyse critique de la notion de variable (points de vue sémiotique et formel), Mathématiques et Sciences humaines, 44e année, $\mathrm{n}^{\circ}$ 173, 43-102.

Desclés, J.-P., Guentchéva, Z. et Shaumyan, S. (1985) : Passivization in Applicative Grammar, John Benjamins Publishing Company.

DesclÉs, J.-P. et GuentChÉVA, Z. (2001): Quantification without Bound Variables, in K. Böttner, W. Thümmel (ed.), Variable free Semantics, Osnabrück, Secolo Verlag, Rolandsmauer, 210233.

Desclés, J.-P. et PAscu, A. (2006): Logic of Determination of Objects: The Meaning of Variable in Quantification, Artificial Intelligence Tools, Architectures, Languages, Algorithms, vol. $15, \mathrm{n}^{\circ} 6,1041-1052$.

DowTy, D., (1979): Word Meaning and Montague Grammar, Dordrecht, D. Reidel Publishing Company.

FrTCH, F.B. (1974) : Elements of Combinatory Logic, New Haven, Yale University Press.

FREGE, G. (1893/1967): Grundgesetze des Arithmetik (1893), traduit en anglais The Basic Laws of Arithmetic, Exposition of a System, Translated and edited, with an Introduction, by Montgrmery Furth, University of California Press. 
FrançOIS, J., (1989): Changement, causation, action, Genève-Paris, Librairie Droz.

FREGE, G. (1971): Ecrits logiques et philosophiques, Paris, Presses universitaires de France.

GuIllaume, G. (1964): Langage et science du langage, Québec, Presses de l'université de Laval.

HARRIS, Z. (1968): Mathematical Structures of Language, New York/London, Interscience Publishers.

HARRIS, Z. (1976) : Notes du cours de syntaxe, Paris, Le Seuil.

HARRIs, Z. (1982) : A Grammar of English on Mathematical Principles, John Wiley \& Sons.

HARris, Z. (1988 / 2007): Language and Information, Columbia University Press, New-York, traduit dans La langue et l'Information, Paris, Cellule de Recherche en linguistique.

Hindley, J. R. et SELDIN, J. (1986): Introduction to Combinators and $\lambda$ Calculus, Cambridge University Press.

Jespersen, O. (1971) : Philosophie de la grammaire, Paris, Minuit.

LAMBEK, J. (1961): On the Calculus of Syntactic Types, in Structure of Language and its Mathematical Aspects, American Mathematical Society, Providence, Rhode Island, 166- 178.

LYONS, J. (1970), Linguistique générale, introduction à la linguistique théorique, Paris, Larousse.

LE Guern, M. (2003) : Les deux logiques du langage, Paris, Honoré Champion.

Mel'Čuk, I. (1987): Dependency Syntax: Theory and Practice, State University of New York Press.

Martin, R. (1992): Pour une logique du sens, Paris, PUF.

Montague, R. (1974 : Formal Philosophy, Selected papers of Richard Montague, edited with an introduction by Richmond $H$. Thomason, New Haven, Yale University Press.

Moorgat, M. (1988): Categorial Investigations, Logical and Linguistic Aspects of the Lambek Calculus, Dordrecht, Foris Publications.

OEHRle, R.T., BACH, E et WheEler, D. (1988): Categorial Grammars and Natural Language Structures, Dordrecht, D. Reidel Publishing Company.

PASCU, A. (2006): Les objets dans la représentation des connaissances. Application aux processus de catégorisation en informatique et dans les Sciences humaines, Dossier d'habilitation à diriger des recherches, Université de Paris-Sorbonne.

PotTIER, B. (1974): Linguistique générale, théorie et description, Paris, Klincksieck. 
POTTIER, B. (1992): Sémantique générale, théorie et description, Paris, Presses universitaires de France.

PotTIER, B. (2000): Représentations mentales et catégorisations linguistiques, Louvain-Paris, Peeters.

Quine, W.V.O. (1970): Philosophy of Logic, Englewood Cliffs, Free Press, New York.

Rousseau, A. (éd.) (1998): La transitivité, Lille, Presses du Septentrion.

RUSSELL, B. (1967): Mathematical Logic as based on the Theory of Types, in J. Van Heijenhoort, From Frege to Gödel, Harvard, Harvard University Press, 150-182.

Shaumyan, S. (1965): Strukturnaya Linguistika, Moscowa, Nauka.

Shaumyan, S. (1987): A Semiotic Theory of Language, Bloomington, Indiana University Press.

Sommers, F. (1982): The Logic of Natural Language, Oxford, Clarendon Press.

Steedman, M. (2000): The Syntactic Process, Cambridge, The MIT Press.

TESNIÈRE, L. (1966) : Éléments de syntaxe structurale, Paris, Klincksieck.

WILMET, M. (1986): La détermination nominale. Quantification et caractérisation, Paris, Presses universitaires de France. 